C-A/AP/\#177

October 2004

\title{
The Effect of the Booster Injection Foil On Emittance
}

K. Zeno

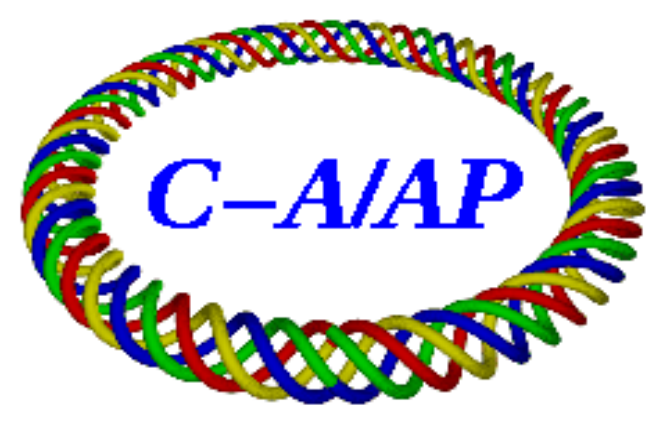

Collider-Accelerator Department Brookhaven National Laboratory Upton, NY 11973 


\section{The Effect of the Booster Injection Foil on Emittance}

K. Zeno

October 31, 2003 


\section{Introduction:}

During polarized proton runs it is important to keep the emittance of the Booster beam at extraction as small as possible. Since, in theory at least, the smaller the transverse emittance, the easier it is to preserve polarization when passing through intrinsic resonances in the AGS. Owing to the fact that the amount of current available from Linac is more than enough to satisfy the intensity requirements in the AGS, a large amount of the beam is often scraped off during acceleration in the Booster to reduce the emittance. Still, it is important to make the beam as small as possible without scraping. Since for a given extraction intensity, the smaller it is without scraping the smaller it will be with it as well.

The amount of beam injected is typically controlled by adjusting the pulse length of the injected beam. The Linac is capable of delivering a pulse width of about 430us. The injection process consists of passing the $\mathrm{H}^{-}$beam coming from Linac through a stripping foil located at $\mathrm{C} 5$ in the Booster ring. The closed orbit is distorted such that it passes through the injection foil. In theory, this injection bump and the trajectory of the incoming proton beam at the foil can be adjusted so that the incoming beam is injected directly onto the closed orbit. Ideally, this configuration would provide the smallest emittance beam.

Booster injection is tuned to minimize the beam size as seen in BtA for a given pulse length. The tuning process is largely a matter of adjusting the injection bump and incoming beam trajectory in order to minimize the beam size in BtA. The Main magnet field is essentially constant during injection, so if the beam trajectory is constant during it, the injection bump should be constant as well to provide the smallest emittance beam. Although there is no control over the trajectory as a function of time in the linac pulse, there is control over the injection bump. In practice, the injection bump that provides the smallest emittance for a 400 us pulse is typically close to constant but not exactly so. It also starts to collapse just after the end of the linac pulse and has collapsed several tens of microseconds later.

During the injection process, the stored beam passes through the foil. Once injection is complete, the injection bump collapses so that the stored beam no longer passes through it. The injected beam's revolution period is $1.2 \mu \mathrm{s}$. So, when the pulse width is $400 \mu \mathrm{s}$, the beam that was injected first has passed through the foil over 300 times. Each time the beam passes through the foil it is scattered causing some emittance increase. If the pulse width is short, the injection bump (or the Linac pulse) can be moved in time such that the bump's collapse begins when the pulse ends, thereby preventing beam from unnecessarily passing through the foil.

To study the effect repeated passing through the stripping foil has on the emittance, a short Linac pulse was used and the injection bump timing was varied. This note examines the dependence of the transverse emittance (as measured in BtA) on the amount of time the injection bump stays on after a short $(80 \mu \mathrm{s})$ Linac pulse has been injected. Then, the 
BtA data is compared to that obtained from a particle tracking model of injection with foil scattering for consistency.

\section{Data}

MW006 and MW060 multiwire profiles were taken for 2 different injection bump timing schemes. MW006 is 6 feet downstream from the beginning of BtA (the F6 septum) and there are no magnetic elements in the line before it. MW060 is 60 feet downstream of it, just past the large bend associated with DH2-3. The first configuration, where the start time of the injection bump (BIJ.FAST.TM) is set to $1270 \mu \mathrm{s}$, has the bump collapsing just as the $80 \mu$ s pulse ends. The other configuration has BIJ.FAST.TM set to $1590 \mu \mathrm{s}$. In this configuration the beam passes through the foil for another $300 \mu \mathrm{s}$ after injection has ended.

In each configuration data was also taken with and without a longitudinal 'bucket squeeze'. This bucket squeeze was used to reduce the Longitudinal bunch width from about $120 \mathrm{~ns}$ to $80 \mathrm{~ns}$ at Booster extraction (as measured on the first turn in AGS). Comparing multiwire data for these cases could show any longitudinal emittance blowup as indicated by an increase in horizontal beam size associated with dispersion effects.

Table I shows the FWHM data for both multiwires and in both planes using the parabolic fit routine. Four sets of data were taken for each of the four cases. Table II shows the data averaged and with standard deviations. The half widths of the beam profiles were also measured by counting the number of wires that encompass the profile, multiplying the result by the wire spacing and dividing by two. This was done for one set of profiles from each of the 16 cases shown in Table I. The results are shown in Table III.

Figure 1 shows a graphical representation of the parabolic fit data. Figure 2 shows a graphical representation of the Table III data obtained by counting wires.

\section{Analysis:}

A glance at Tables 1 and 2 shows a rather clear dependence of the vertical beam size on injection bump timing for both multiwires. That is, the $1590 \mu$ s case is always wider than the $1270 \mu$ s case. However, the horizontal dependence is not so clear. Yet, it seems likely that this may be due in part to limitations in the measurements. At MW006 the Horizontal Beta function is small so the horizontal beam is quite narrow and the parabolic fit does not do a good job fitting it as can be seen in figure 1. Additionally, because the beam is so narrow there, the resolution of the counting wires method for MW006 in the Horizontal plane is not sufficient to always show changes in width that are relevant. Also, the baseline for the horizontal MW060 profile is ragged and it is difficult to measure the size of the profile using the counting wire method in this case (figure 2). But, the parabolic fit does a reasonable job for the horizontal profile at MW060 (see figure 2). So, the parabolic fit data for H MW060 are the most reliable of the four and this data does show a clear dependence. 


\begin{tabular}{|c|c|c|c|c|}
\hline & & t squeeze & & Squeeze \\
\hline & $(15$ & (1270us) & $(159)$ & (1270us) \\
\hline & 7.38 & 6.57 & 6.69 & 6.61 \\
\hline MW006 & 6.62 & 6.61 & 6.62 & 6.61 \\
\hline Horiz. & 7.39 & 6.58 & 6.66 & 6.49 \\
\hline & 7.34 & 6.59 & 6.68 & 6.60 \\
\hline & 12.50 & 9.49 & 12.49 & 9.53 \\
\hline MW006 & 11.66 & 9.61 & 12.17 & 9.47 \\
\hline Vert. & 12.39 & 9.61 & 11.38 & 9.21 \\
\hline & 11.68 & 9.60 & 12.52 & 9.58 \\
\hline & 25.05 & 21.74 & 25.82 & 21.68 \\
\hline MW060 & 24.70 & 22.52 & 22.83 & 20.65 \\
\hline Horiz. & 25.47 & 22.78 & 25.66 & 20.94 \\
\hline & 25.71 & 22.77 & 23.20 & 19.43 \\
\hline MW060 & 13.38 & 11.11 & 14.13 & 10.89 \\
\hline Vert & $\begin{array}{l}1 \\
3.32\end{array}$ & 12.34 & 12.79 & 11.00 \\
\hline & 13.45 & 12.25 & 13.99 & 10.89 \\
\hline & 13.54 & 11.21 & 12.60 & 11.01 \\
\hline
\end{tabular}

Table I: Full Width at Half Maximum (FWHMs) of multiwire profile data using parabolic fitting routine from BeamLineInstrument, in $\mathrm{mm}$.

\begin{tabular}{|l|l|l|l|l|}
\hline & \multicolumn{2}{|c|}{$\begin{array}{c}\text { No Bucket squeeze } \\
(\mathbf{1 5 9 0 u s})\end{array}$} & \multicolumn{2}{c|}{$\begin{array}{c}\text { Bucket Squeeze } \\
(\mathbf{1 2 7 0 u s})\end{array}$} \\
\hline MW006 & 7.2 & 6.6 & 6.7 & 6.6 \\
Horiz. & $\sigma=0.33$ & $\sigma=0.04$ & $\sigma=0.03$ & $\sigma=0.05$ \\
\hline MW006 & 12.1 & 9.6 & 12.1 & 9.4 \\
Vert. & $\sigma=0.39$ & $\sigma=0.05$ & $\sigma=0.46$ & $\sigma=0.14$ \\
\hline MW060 & 25.2 & 22.5 & 24.4 & 20.7 \\
Horiz. & $\sigma=0.39$ & $\sigma=0.42$ & $\sigma=1.37$ & $\sigma=0.81$ \\
\hline MW060 & 13.4 & 11.7 & 13.4 & 10.9 \\
Vert & $\sigma=0.08$ & $\sigma=0.57$ & $\sigma=0.69$ & $\sigma=0.06$ \\
\hline
\end{tabular}

Table II: Averages and standard deviations of the FWHM data in Table I, in mm.

\begin{tabular}{|l|l|l|l|l|}
\hline & \multicolumn{2}{|c|}{$\begin{array}{c}\text { No Bucket Squeeze } \\
(1590 u s)\end{array}$} & \multicolumn{2}{c|}{$\begin{array}{c}\text { Bucket Squeeze } \\
\text { (1270us) }\end{array}$} \\
\hline $\begin{array}{l}\text { MW006 } \\
\text { Horiz. }\end{array}$ & 8 & 8 & 8 & 8 \\
\hline $\begin{array}{l}\text { MW006 } \\
\text { Vert. }\end{array}$ & 14 & 12 & 13 & 12 \\
\hline $\begin{array}{l}\text { MW060 } \\
\text { Horiz. }\end{array}$ & 17 & 17 & 17 & 14 \\
\hline $\begin{array}{l}\text { MW060 } \\
\text { Vert }\end{array}$ & 10 & 8 & 10 & 7 \\
\hline
\end{tabular}

Table III: Number of wires obtained from counting wires in multiwire profiles. MW006 wires are $1.5 \mathrm{~mm}$ apart, MW060 wires are $2.5 \mathrm{~mm}$ apart. Each case has one set of data. 


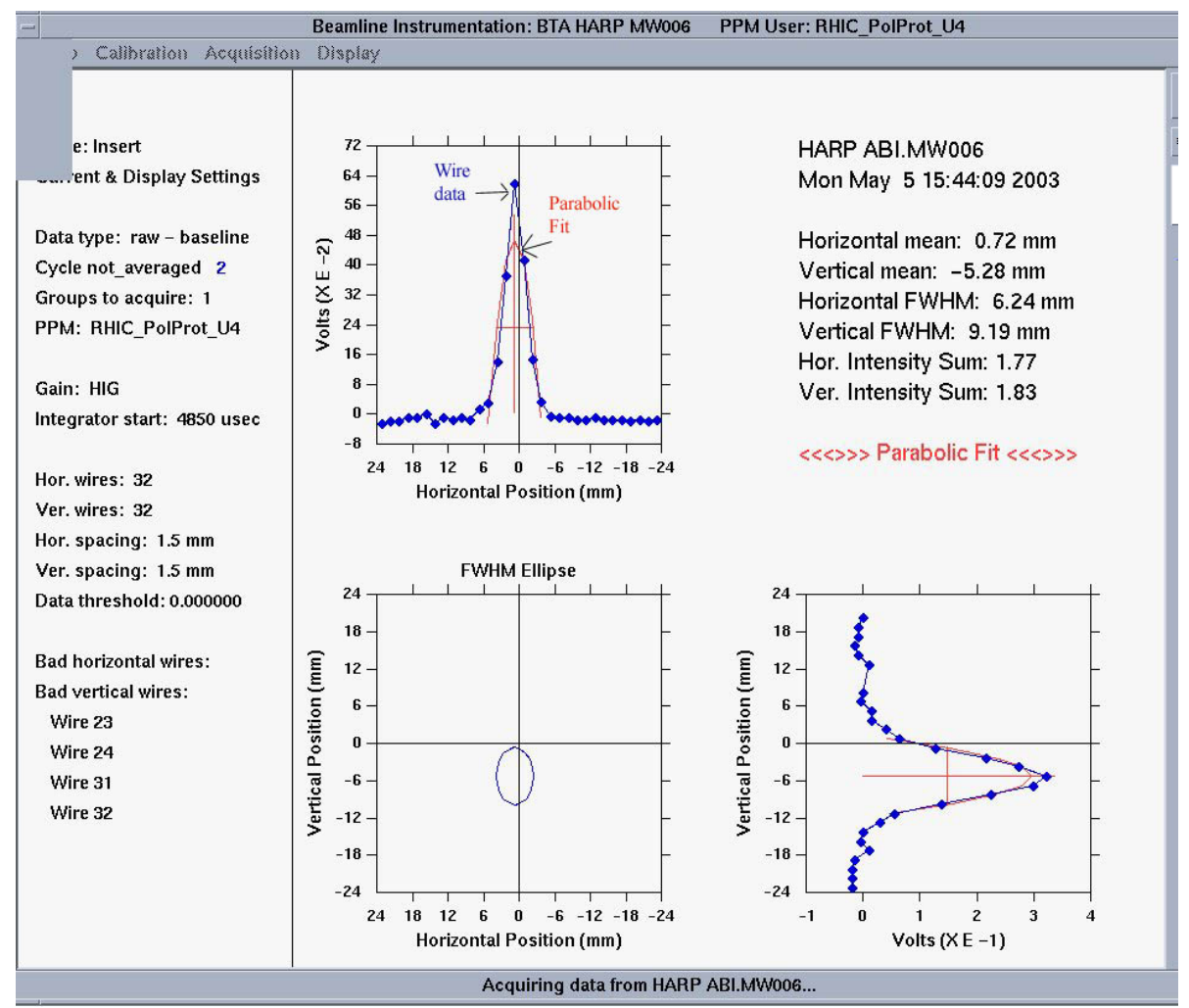

Figure 1: MW006 profiles showing parabolic fitting.

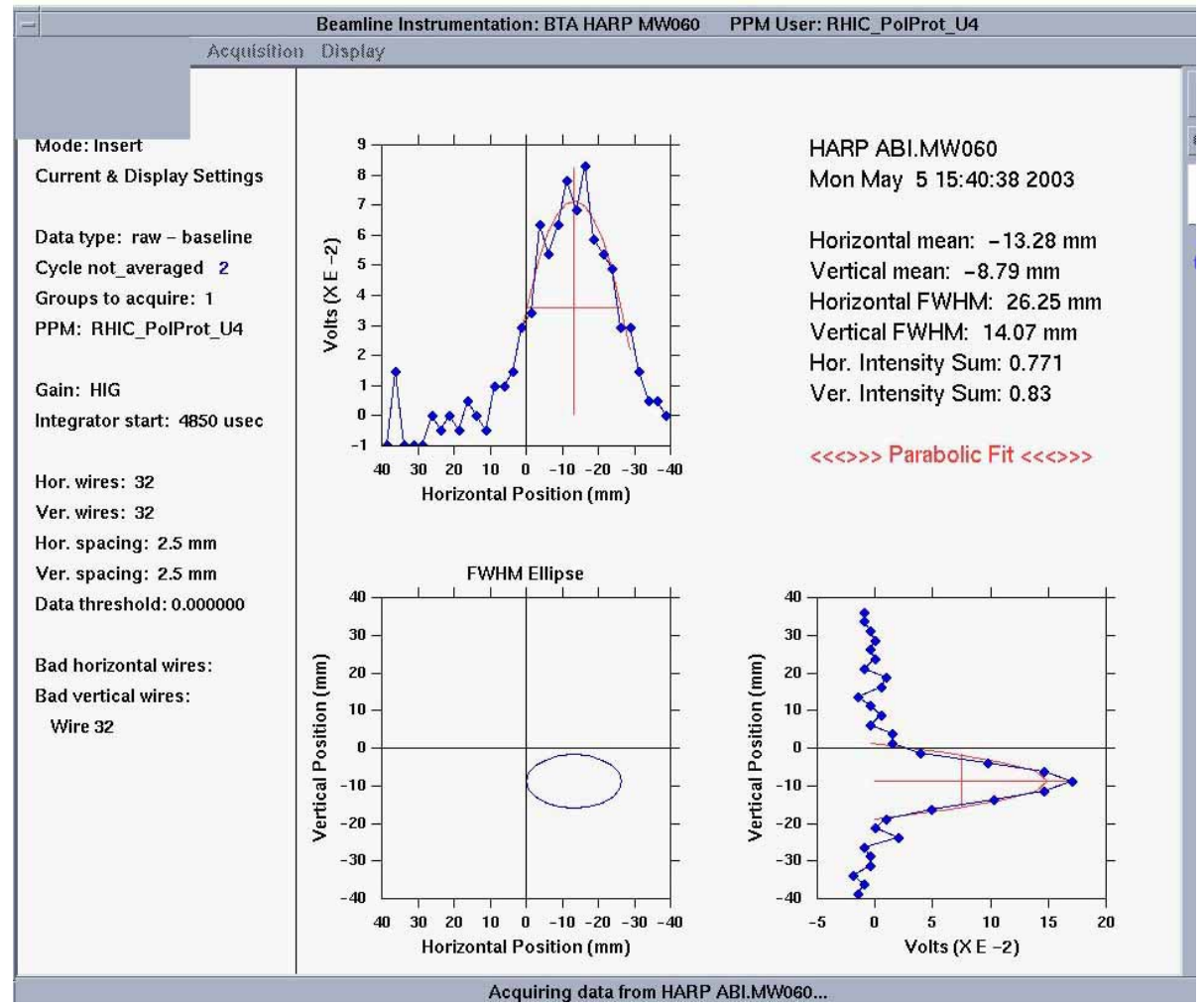

Figure 2: MW060 data showing ragged horizontal baseline and parabolic fitting. 
In the vertical plane, the data for MW006 is more reliable than that for MW060 since the beam is nearly the same size on both multiwires and there are more wires on MW006. For the vertical MW006 data the parabolic fit method is probably preferable since it removes the subjectivity that is inherent in the counting wires method.

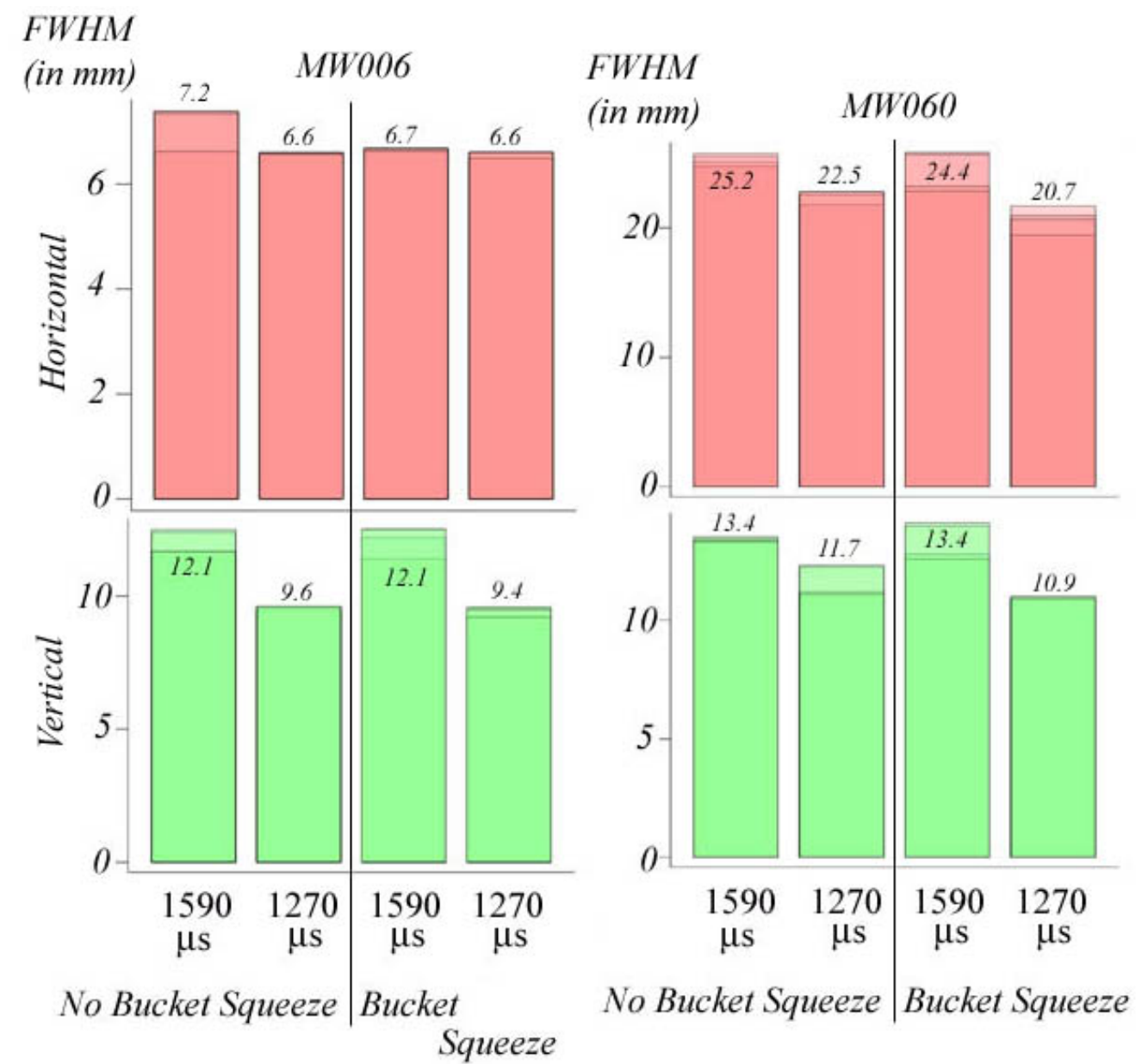

Figure 3: Full widths at half maximum (FWHMs) from parabolic fit data in Tables I and II. For each of the 16 cases, the four sets of data are overlayed. The average values for the FWHMs are shown at the top of each bar.

When there is a bucket squeeze occuring the data should be more indicative of the actual change in transverse emittance in the horizontal plane. So, using this data, the horizontal width on MW060 increases by a factor of 25.2/22.5=1.12. In the vertical, the MW006 width increases by a factor of $12.1 / 9.6=1.26$. In terms of emittance, $\varepsilon_{\mathrm{V}}=1.26^{2}=1.59$, $\varepsilon_{\mathrm{h}}=1.12^{2}=1.25$. 


\section{Particle Tracking Model}

A particle tracking model was developed using Mathcad in order to find a consistent picture that would explain the observed emittance increase associated with multiple passes through the foil, as well as the emittances measured in BtA. The following assumptions and simplifications were made to make the process manageable:

1) There is no emittance increase after the end of the injection process.

2) In the model, all the beam is injected on one turn instead of the actual 67 turns ( 80 us). The model then tracks these particles for 284 turns $=340 \mu \mathrm{s}$. In the Booster, the first turn injected passes through the foil somewhere around 300 turns and the last turn injected 60-70 turns less than that.

3) The change in emittance observed from that of the Linac beam to that measured in BtA is solely due to mismatching at injection and multiple passes through the foil.

4) Since the values of the incoming beam's Twiss parameters, the dispersion at the foil, momentum spread, and transverse emittance are not precisely known, they were varied in the model over a 'reasonable' range to find the most consistent picture.

5) The scattering angles of the beam passing through the foil are treated as having a Gaussian distribution. The standard deviation of the distribution is varied to fit the observed BtA data.

6) The momentum and tranverse distributions of the linac beam are treated as Gaussian.

7) The Lattice parameters for the Booster at the foil are those given by the MAD model.

8) For each case, the resulting emittance values use at least 2010 particles.

In the model, the initial $\left(x, x^{\prime}\right)$ and $\left(y, y^{\prime}\right)$ coordinates for the $i$ th particle are derived from a gaussian distribution by the following,

$$
\begin{aligned}
& x_{i}=A \sqrt{k_{i} \beta_{x}} \cos \left(\phi_{i}\right) \\
& x_{i}^{\prime}=\frac{-A \sqrt{k_{i}}}{\sqrt{\beta_{x}}}\left[\alpha_{x} \cos \left(\phi_{i}\right)+\sin \left(\phi_{i}\right)\right] \\
& y_{i}=A \sqrt{k_{i} \beta_{y}} \cos \left(\phi_{i}\right) \\
& y_{i}^{\prime}=\frac{-A \sqrt{k_{i}}}{\sqrt{\beta_{y}}}\left[\alpha_{y} \cos \left(\phi_{i}\right)+\sin \left(\phi_{i}\right)\right]
\end{aligned}
$$

where $k_{i}$ are the absolute values of elements of a gaussian distribution centered around zero with $\sigma=0.2$. A is a constant that sets the overall amplitude. $\phi_{\mathrm{i}}$ are uniform random 
numbers between 0 and $2 \pi . \beta_{x}, \alpha_{x}, \beta_{y}$, and $\alpha_{y}$ are variables that represent the Twiss parameters of the beam at the foil.

The effect of the $j$ th pass through the foil on the $i$ th particle is modeled by replacing the $x_{i, j}^{\prime}$ with $x_{i, j}^{\prime}+\varphi_{i, j}$, where for each $j, \varphi_{i, j}$, is an element of a gaussian distribution centered on zero with the specified $\sigma$. So, $\varphi_{i, j}$ represents the scattering angle in milliradians that the $i$ th particle receives on the $j$ th pass through the foil.

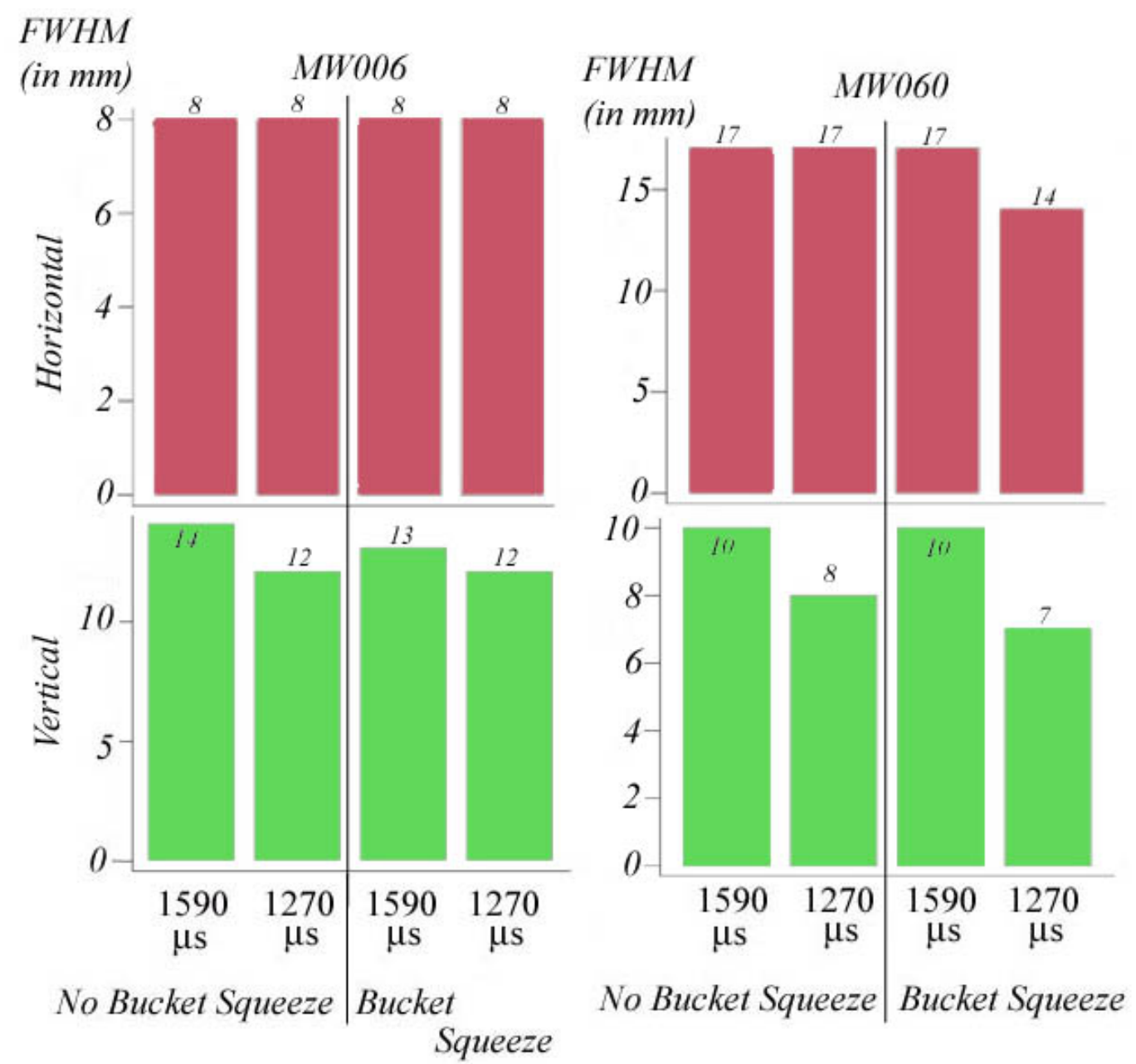

Figure 4: Data using counting wires method from Table III. MW006 wires are 1.5mm apart, MW060 wires are $2.5 \mathrm{~mm}$ apart. Only one set of data taken for each case. The values for each case are shown at the top of each bar.

For each of the $j$ passes, $\left(x_{i, j}, x_{i, j}^{\prime}\right)$ is multiplied by the $2 \times 2$ one turn transfer matrix $M=I \cos \psi+J \sin \psi$ to simulate one turn around the ring, where $I$ is the $2 \mathrm{x} 2$ identity matrix,

$$
J=\left[\begin{array}{cc}
\alpha & \beta \\
-\gamma & -\alpha
\end{array}\right],
$$


and $\psi$ is the phase advance around the ring $(2 \pi v)$, where $v$ is in general taken as 4.80 . The parameters $\alpha, \beta$, and $\gamma$ are the lattice parameters at the foil given by MAD.This is repeated until $j=T$, where $T$ is the number of passes through the foil for each of the $N$ particles and has been set to 284. This description is for the $x$-plane, but the same holds true for the $y$-plane.

The initial beam emittance, $\varepsilon_{\mathrm{i}}$, is found by calculating the particle emittance for each of the 2010 (or more) particles before passing through the foil and using the foil's lattice parameters $\left(\beta_{\mathrm{x}}=10.852 \mathrm{~m}, \alpha_{\mathrm{x}}=-1.571, \beta_{\mathrm{y}}=4.751 \mathrm{~m}, \alpha_{\mathrm{y}}=0.851\right)$. The beam emittance is then taken as the area of the ellipse defined by the foil's lattice parameters with an area that encloses $95 \%$ of these particles. The final beam emittance, $\varepsilon_{\mathrm{f}}$ is calculated the same way except that the particle emittances after 284 'passes' through the foil are used. These emittances are normalized by multiplying by $\beta \gamma=0.68$.

Figure 5 shows $\varepsilon_{\mathrm{f}}$ as a function of $\varepsilon_{\mathrm{i}}$ for $\sigma=0.03$ and $0.05 \mathrm{mrad}$ for matched injection. The BtA emittances are also shown. The 'average' BtA emittances are derived from the counting wires method using MW006. For the horizontal, the number of wires is 8 for all cases (figure 4). The horizontal emittance is $\left.(8 \text { wires* } 1.5 \mathrm{~mm} / 2)^{2} / \beta_{\mathrm{x}}\right)^{*} \beta \gamma=\left(36 \mathrm{~mm}^{2} / 4.2\right.$ $m$ ) $2.4=21 \pi \mathrm{mm} \mathrm{mrad}$ (see Table III). For the vertical emittance the average value of 12.75 wires is used (figure 4$)$. This value yields, $(12 \text { wires } * 1.5 \mathrm{~mm} / 2)^{2} / \beta_{\mathrm{y}}{ }^{*} \beta \gamma=$ $\left(91.4 \mathrm{~mm}^{2} / 17.85 \mathrm{~m}\right)^{*} 2.4=12.3 \pi \mathrm{mm} \mathrm{mrad}$. Using MW006 data is preferable because $\beta$ is better known there than it is at MW060.

One characteristic of the data is its linearity. This is likely due to fact that the one turn transfer matrix is linear. Linear fitting of the data shows that the only obvious difference between the 4 sets of data are their $y$-intercepts. In particular, the slopes for $\sigma=0.03 \mathrm{mrad}$ and $\sigma=0.05 \mathrm{mrad}$ are the equal, as are the slopes for the horizontal and vertical cases. The linearity is also an indication that the number of data sets (2010) is sufficient to give an accurate picture of the effect of the foil.

Given that the measured horizontal $\varepsilon_{\mathrm{f}} / \varepsilon_{\mathrm{i}}$ for MW060 is 1.25 , a value for the horizontal emittance with and without blow-up from the foil can be estimated, assuming that the emittance increase is linear with the number of passes through the foil, and that the emittance measured at MW006 is its average value $\left(\varepsilon_{\mathrm{avg}}=21 \pi \mathrm{mm} \mathrm{mrad}\right) .{ }^{1}$ Then, $\left(\varepsilon_{\mathrm{f}}+\varepsilon_{\mathrm{i}}\right) / 2=\left(2.25 \varepsilon_{\mathrm{i}}\right) / 2=\varepsilon_{\mathrm{avg}}$, so $\varepsilon_{\mathrm{i}}=(2 / 2.25) \varepsilon_{\mathrm{avg}}=18.7 \pi \mathrm{mm} \mathrm{mrad}$, and similarly $\varepsilon_{\mathrm{f}}=23.3 \pi$ $\mathrm{mm} \mathrm{mrad}$. In the vertical plane, $\left(\varepsilon_{\mathrm{f}}+\varepsilon_{\mathrm{i}}\right) / 2=\left(2.59 \varepsilon_{\mathrm{i}}\right) / 2=\varepsilon_{\mathrm{avg}}=12.3 \pi \mathrm{mm} \mathrm{mrad}$ so $\varepsilon_{\mathrm{i}}=(2 / 2.59) \varepsilon_{\mathrm{avg}}=9.5 \pi \mathrm{mm} \mathrm{mrad}$, and $\varepsilon_{\mathrm{f}}=15.1 \pi \mathrm{mm} \mathrm{mrad}$. These $\varepsilon_{\mathrm{f}}$ and $\varepsilon_{\mathrm{i}}$ values are the BtA values shown in figure 5 .

Figure 7 shows curves derived from the linear fits of the data in figure 5 . The curves were obtained by dividing by both sides of the linear fit equations by $\varepsilon_{\mathrm{i}}$. That is,

\footnotetext{
${ }^{1}$ Figure 6 shows $\varepsilon_{f} / \varepsilon_{i}$ from particle tracking as a function of the number of turns. This data shows that the assumption that the emittance increase is linear is a reasonable one.
} 


$$
\varepsilon_{f}=m \varepsilon_{i}+b \rightarrow \frac{\varepsilon_{f}}{\varepsilon_{i}}=b \frac{1}{\varepsilon_{i}}+m
$$

Since the figure 5 data is linear there is an inverse relationship between the initial emittance and $\varepsilon_{\mathrm{f}} / \varepsilon_{\mathrm{i}}$. The average slope of the 4 sets of data is 0.977 . This is consistent with the idea that, as the initial emittance becomes very large, the emittance blow-up from the foil becomes negligible since $\varepsilon_{\mathrm{f}} / \varepsilon_{\mathrm{i}}$ approaches one.

From figure 7 it can be seen that the model expects the foil's effect to be more pronounced in the horizontal (red) than in the vertical (blue) plane, although the measured $\varepsilon_{\mathrm{f}} / \varepsilon_{\mathrm{i}}$ in $\mathrm{BtA}$ has the opposite sense. That is, if the vertical $\varepsilon_{\mathrm{i}}$ were the same as the horizontal then the emittance blow-up expected would be much less than for the horizontal, and vice-versa. The points shown from BtA are not strictly $\left(\varepsilon_{i}, \varepsilon_{f}\right)$ but are what the beam looks like in BtA whether the beam passes through the foil a lot or a little. Other factors, such as injection mismatching could explain why the horizontal " $\varepsilon_{\mathrm{i}}$ " is much larger than the vertical " $\varepsilon_{\mathrm{i}}$ " even though the beam coming into the Booster might have the same emittance in each plane.

Using a model of the Twiss parameters at LtB MW035 and counting wires on that multiwire gives normalized $95 \%$ emittance $\varepsilon_{\mathrm{x}}$ of $6 \pi \mathrm{mm} \mathrm{mrad}$, and $\varepsilon_{\mathrm{y}}=5 \pi \mathrm{mm} \mathrm{mrad}{ }^{2}$ Figure 7 shows that the horizontal emittance would have to be unrealistically large for $\sigma=0.05(>30 \pi \mathrm{mm} \mathrm{mrad})$ in order for $\varepsilon_{\mathrm{f}} / \varepsilon_{\mathrm{i}}$ to be that observed in BtA. Alternately, if $\sigma=0.03$, than the vertical would have to be unrealistically small $(<2.5 \pi \mathrm{mm} \mathrm{mrad}$ for $\sigma=0.03$ ). The nominal value given for the Linac beam emittance is about $10 \pi \mathrm{mm} \mathrm{mrad}$.

So, the observed " $\varepsilon_{\mathrm{i}}$ " in BtA for the horizontal is much larger than one expects if the injected beam is matched to the Booster $(18.5 \pi \mathrm{mm} \mathrm{mrad}$ vs. $<10 \pi \mathrm{mm} \mathrm{mrad})$. Whereas the vertical " $\varepsilon_{i}$ " is in much better agreement (about $11 \pi \mathrm{mm} \mathrm{mrad}$ vs. $<10 \pi \mathrm{mm} \mathrm{mrad}$ ). Also, the vertical $\varepsilon_{\mathrm{f}} / \varepsilon_{\mathrm{i}}$ for $\sigma=0.05 \mathrm{mrad}$ and $\varepsilon_{\mathrm{i}} \sim 6.9 \pi \mathrm{mm} \mathrm{mrad}$, a reasonable value, is the same as the vertical $\varepsilon_{\mathrm{f}} / \varepsilon_{\mathrm{i}}$ measured in BtA. Although, obviously, many other possibilites exist, if $\sigma$ was $0.05 \mathrm{mrad}$, other processes, such as transverse coupling could possibly account for the the emittance growth from 6.9 to $9.5 \pi \mathrm{mm} \mathrm{mrad}$ during the cycle. ${ }^{3}$ Changing the tunes during the cycle has an effect on the relative horizontal and vertical emittances in BtA. These tunes are adjusted to minimize this 'coupling'. Since the tunes, at least in the BoosterTuneControl application do not cross, the indication that coupling has been minimized is that the vertical BtA emittance is minimized, and the horizontal BtA emittance is maximized. However, some small amount of coupling may still be present.

\footnotetext{
${ }^{2}$ K. Brown, LtB MAD calculation.

${ }^{3}$ It's also likely that the model, due to the simplifying assumptions made in it, cannot account for discrepancies at this level.
} 


\section{Final Emittance as a Function of Initial Emittance for Scattering Angle $\sigma$ of 0.03 and $0.05 \mathrm{mrad}$}

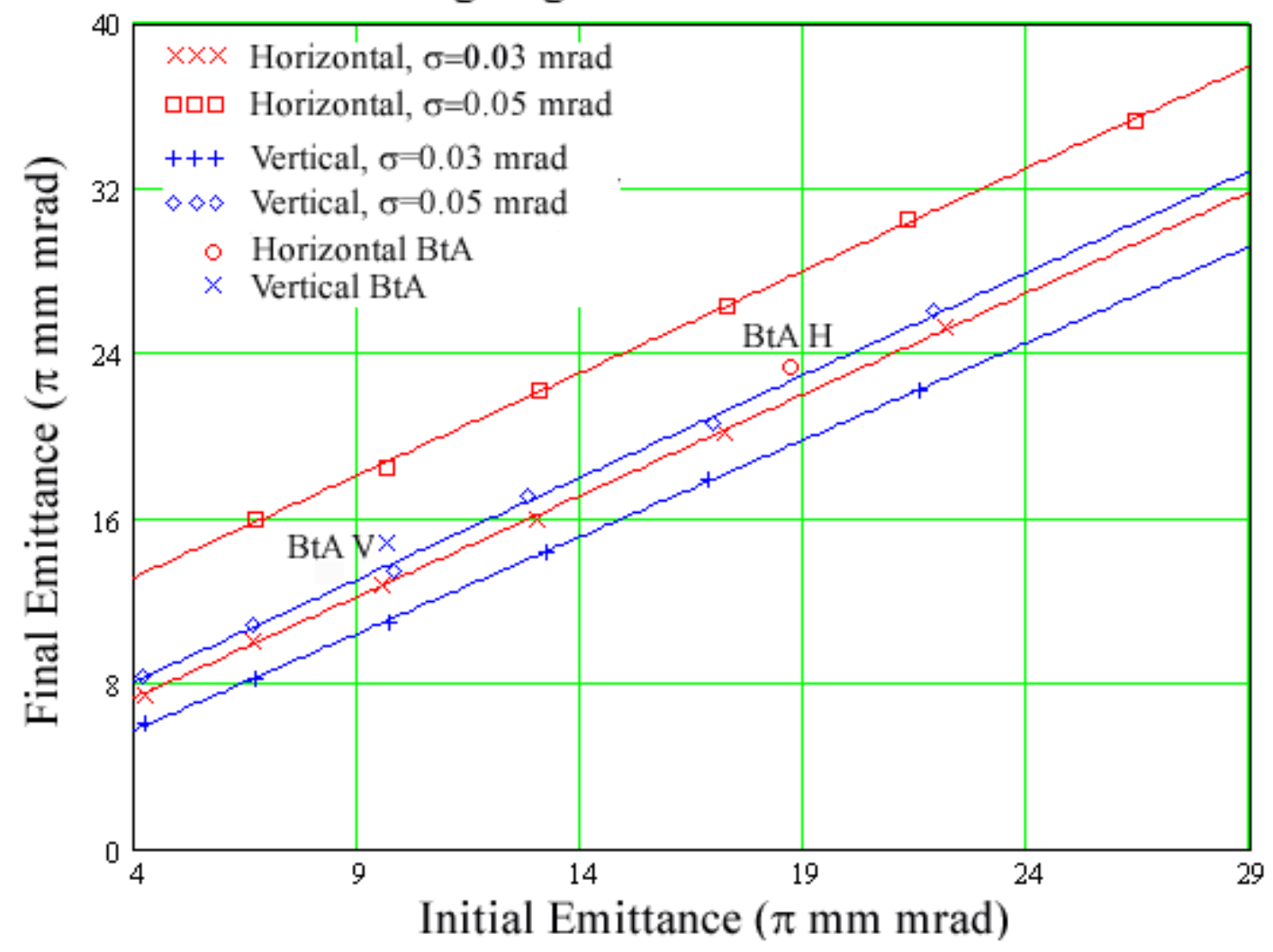

Figure 5: Initial versus final $95 \%$ normalized emittances for $\sigma=0.03$ and $0.05 \mathrm{mrad}$. Measured BtA emittance values are also shown, as well as linear fits of the data.

So, the behavior of the vertical profile in BtA is rather consistent with the $\sigma=0.05 \mathrm{mrad}$ case, the problem is how to make the horizontal profile consistent with this case since the scattering must be the same in either plane. It may be that mismatching will have a similar effect to increasing the incoming emittance on $\varepsilon_{f} / \varepsilon_{i}$. That is, it could explain why the horizontal $\varepsilon_{\mathrm{f}} / \varepsilon_{\mathrm{i}}$ is less than the vertical $\varepsilon_{\mathrm{f}} / \varepsilon_{\mathrm{i}}$, while at the same time explaining why the horizontal emittance is larger than expected. With this in mind, the effect of mismatching injection horizontally on $\varepsilon_{\mathrm{f}} / \varepsilon_{\mathrm{i}}$ and $\varepsilon_{\mathrm{i}}$ was investigated.

In the case of a trajectory mismatch the $x$ coordinate of the $i$ th incoming particle, $x_{i}$, is replaced with $x_{i}+\Delta x$, where $\Delta x$ has the same value for all incoming particles. A dispersion mismatch is like a trajectory mismatch, except $\Delta x$ is not a constant. In that case, $\Delta x_{i}=\Delta D(\Delta p / p)_{i}$. Where $(\Delta p / p)_{i}$ is the momentum offset of the $i$ th particle. In the model, the momentum offset distribution is Gaussian and the $\sigma$ of the distribution can be varied. The term $\Delta D$ is equal to $D_{l t b}-D_{\text {Booster }}$, where $D_{l t b}$ is the dispersion of the incoming beam at the foil, and $D_{\text {Booster }}$ is the dispersion of the Booster at the foil. So, for a dispersion 
mismatch, the $\Delta x_{i}$ is added to the $x$ coordinate of the $i$ th incoming particle (that is, $\left.x_{i}->x_{i}+\Delta x_{i}\right)$.

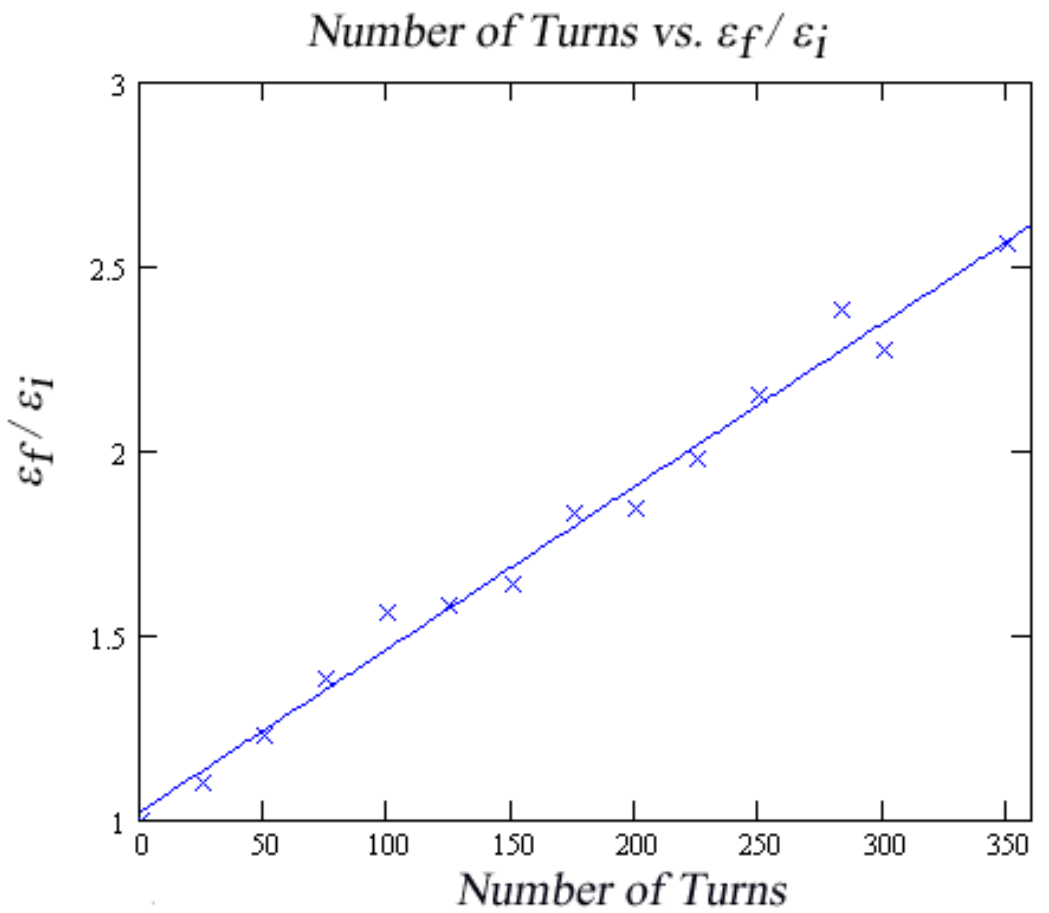

Figure 6: Number of passes through the foil vs. horizontal emittance increase $\left(\varepsilon_{\mathrm{f}} / \varepsilon_{\mathrm{i}}\right)$. The data is consistent with using $\left(\varepsilon_{\mathrm{f}}+\varepsilon_{\mathrm{i}}\right) / 2$ as an approximation for the average emittance.

In the case of a $\beta_{x}$ mismatch, the $\beta_{x}$ of the incoming $x_{i}$, in eq. (1), which is nominally the same as the Booster's $\beta_{\mathrm{x}}$ at the foil $\left(=\beta_{\text {Booster }}\right)$, is replaced with a different value $\left(=\beta_{l t b}\right)$. The $\beta$ function mismatch is described by $\Delta \beta=\beta_{l t b}-\beta_{\text {Booster. }}$. The $x$ ' coordinate of the $i$ th incoming particle is chosen randomly from the two roots of the particle emittance equation,

$$
\varepsilon_{i}=\gamma_{l t b} x_{i}^{2}+2 \alpha_{l t b} x_{i} x_{i}^{\prime}+\beta_{l t b} x_{i}^{\prime 2}
$$

when solved for $x^{\prime}{ }_{i}$, where $\varepsilon_{i}=A \sqrt{k_{i}}$ as in eq. (1). $\alpha$ could be mismatched just as well, but was typically set equal to its value in the Booster.

Figure 8 shows the dependence of $\varepsilon_{\mathrm{f}}$ on $\varepsilon_{\mathrm{i}}$ in the cases of $\beta$ function and dispersion mismatch in the horizontal plane for $\sigma=0.05 \mathrm{mrad}$. $\varepsilon_{\mathrm{i}}$ is the area of the Booster's phase space ellipse at the foil which encloses $95 \%$ of the beam (normalized for injection energy). So, it already includes blow-up from the mismatching. Whether the dispersion or the $\beta$ function is mismatched, the dependence of $\varepsilon_{\mathrm{f}}$ on $\varepsilon_{\mathrm{i}}$ is essentially the same. Also, shown are a dispersion mismatch cases $\sigma=0.03 \mathrm{mrad}$ and $0.07 \mathrm{mrad}$. The main difference between these cases and the $\sigma=0.05 \mathrm{mrad}$ case is their y-intercepts. 
$\varepsilon_{\mathrm{f}} / \varepsilon_{\mathrm{i}}$ as a Function of Initial Emittance

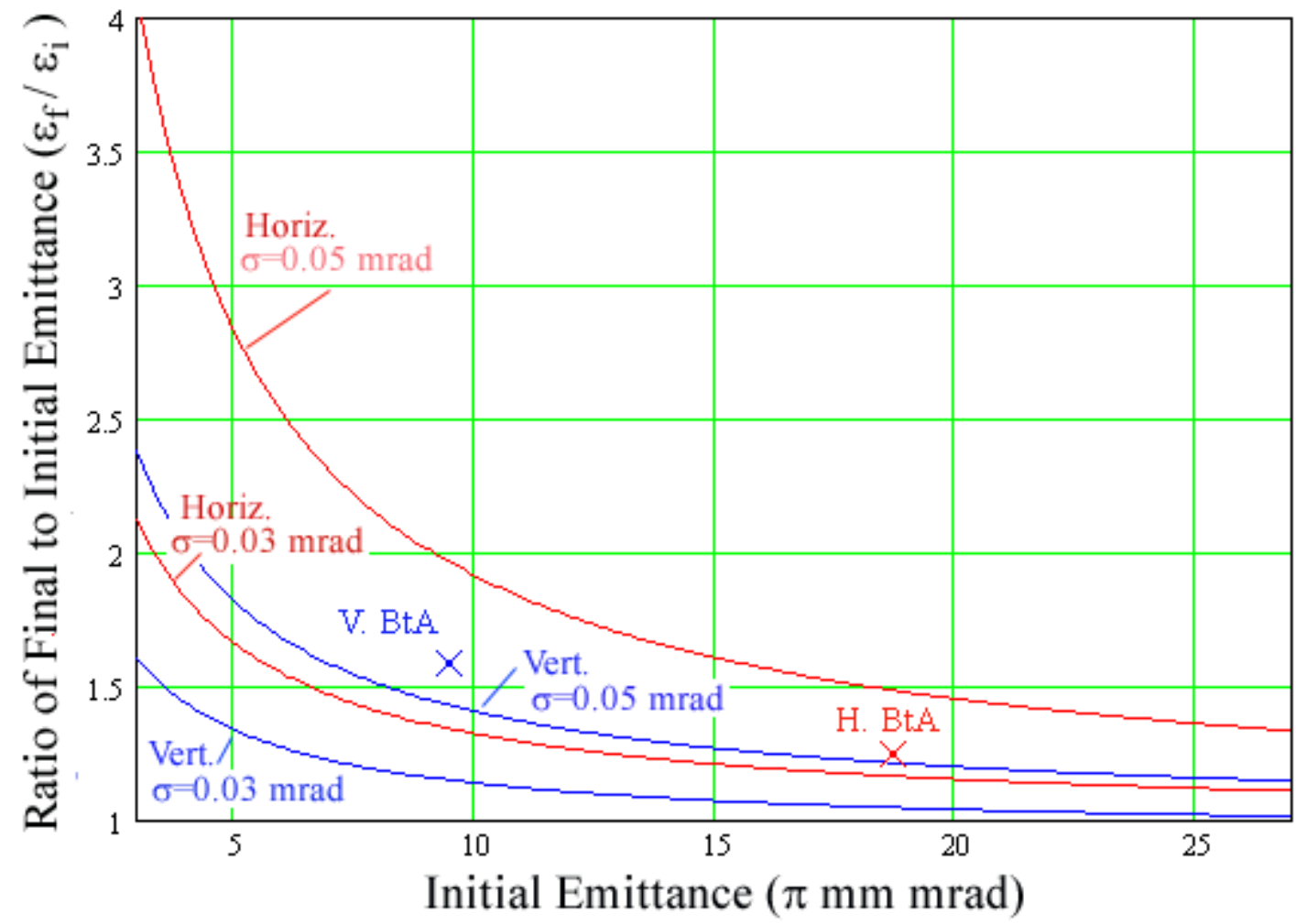

Figure 7: $\varepsilon_{\mathrm{f}} / \varepsilon_{\mathrm{i}}$ as a function of initial emittance for $\sigma=0.03$ and $0.05 \mathrm{mrad}$. The curves are derived from the linear fits of the data in figure 5. The BtA data is also shown.

Figure 9 shows $\varepsilon_{\mathrm{f}} / \varepsilon_{\mathrm{i}}$ as a function of $\Delta \beta$ and $\Delta \mathrm{D}$. In both cases, if the mismatch is large enough, $\varepsilon_{\mathrm{f}} / \varepsilon_{\mathrm{i}}$ will attain a value of 1.25 . However, in all cases it does not drop that low before $\varepsilon_{\mathrm{i}}$ has become significantly larger than what is seen in $\mathrm{BtA}$. The dispersion mismatch $\sigma_{\mathrm{p}}=0.12 \%$ case comes closest to satisfying $\varepsilon_{\mathrm{f}} / \varepsilon_{\mathrm{i}}=1.25$ for the horizontal $\varepsilon$ observed in BtA (21 $\pi \mathrm{mm} \mathrm{mrad})$. In that case, the average horizontal $\varepsilon,\left(\varepsilon_{\mathrm{f}}+\varepsilon_{\mathrm{i}}\right) / 2$, reaches $21 \pi \mathrm{mm}$ mrad around $\Delta \mathrm{D}=3 \mathrm{~m}$. At $\Delta \mathrm{D}=3 \mathrm{~m}, \varepsilon_{\mathrm{f}} / \varepsilon_{\mathrm{i}}$ is about 1.34 . Whereas, $\varepsilon_{\mathrm{f}} / \varepsilon_{\mathrm{i}}$ reaches 1.25 around $\Delta \mathrm{D}=4 \mathrm{~m}$, where the average $\varepsilon$ is about $30 \pi \mathrm{mm} \mathrm{mrad}$. However, in figure 7 the $\sigma=0.05 \mathrm{mrad}$ linear fits essentially overlap, so the difference between the approach of the 4 cases to what is seen in BtA is likely due to lack of statistics.

Figure 10 shows the figure 8 linear fit data in terms of $\varepsilon_{\mathrm{f}} / \varepsilon_{\mathrm{i}}$ vs. $\varepsilon_{\mathrm{i}}$ (similar to figure 5). The $\sigma=0.05 \mathrm{mrad}$ curve is derived from a linear fit of all the $\sigma=0.05 \mathrm{mrad}$ data. The figure also shows data for the vertical plane. Assuming the emittance behavior of the vertical beam is solely due to the foil passage, a $\sigma$ greater than $0.05 \mathrm{mrad}$, but clearly less than $0.07 \mathrm{mrad}$ would be required to obtain the observed $\varepsilon_{\mathrm{f}} / \varepsilon_{\mathrm{i}}$ of 1.59 . However, $\sigma=0.05 \mathrm{mrad}$ gives an answer that is rather close, and a larger $\sigma$ requires a larger horizontal $\varepsilon_{\mathrm{i}}$ to satisfy the horizontal $\varepsilon_{\mathrm{f}} / \varepsilon_{\mathrm{i}}$ requirement of 1.25 . The $\varepsilon_{\mathrm{i}}$ for $\sigma=0.05 \mathrm{mrad}$ and $\varepsilon_{\mathrm{f}} / \varepsilon_{\mathrm{i}}=1.25$ is already larger than that observed in BtA. So, for the horizontal, a larger $\sigma$ would only increase the gap between what's seen in BtA and what the model indicates. 
Since the effect of the foil is greater in the horizontal if the beam is matched, it does not matter as much if the beam is mismatched because the mismatch reduces the amount of emittance blow-up. From figure 9, if the beam had no dispersion or $\beta$ function mismatch, and $\sigma=0.05 \mathrm{mrad}$, the horizontal $\varepsilon_{\mathrm{f}} / \varepsilon_{\mathrm{i}}$ would be 2.4 . With an initial emittance the same as the vertical $(6.89 \pi \mathrm{mm} \mathrm{mrad})$, the final emittance would be $16.5 \pi \mathrm{mm} \mathrm{mrad}$. Although these emittances are less than what are observed in BtA, (i.e., $\left(\varepsilon_{i}, \varepsilon_{\mathrm{f}}\right)=(18,23) \pi \mathrm{mm}$ $\mathrm{mrad}$ ), the benefit of matching on the emittance is significantly offset by the reduction in emittance blow-up afforded by mismatching.

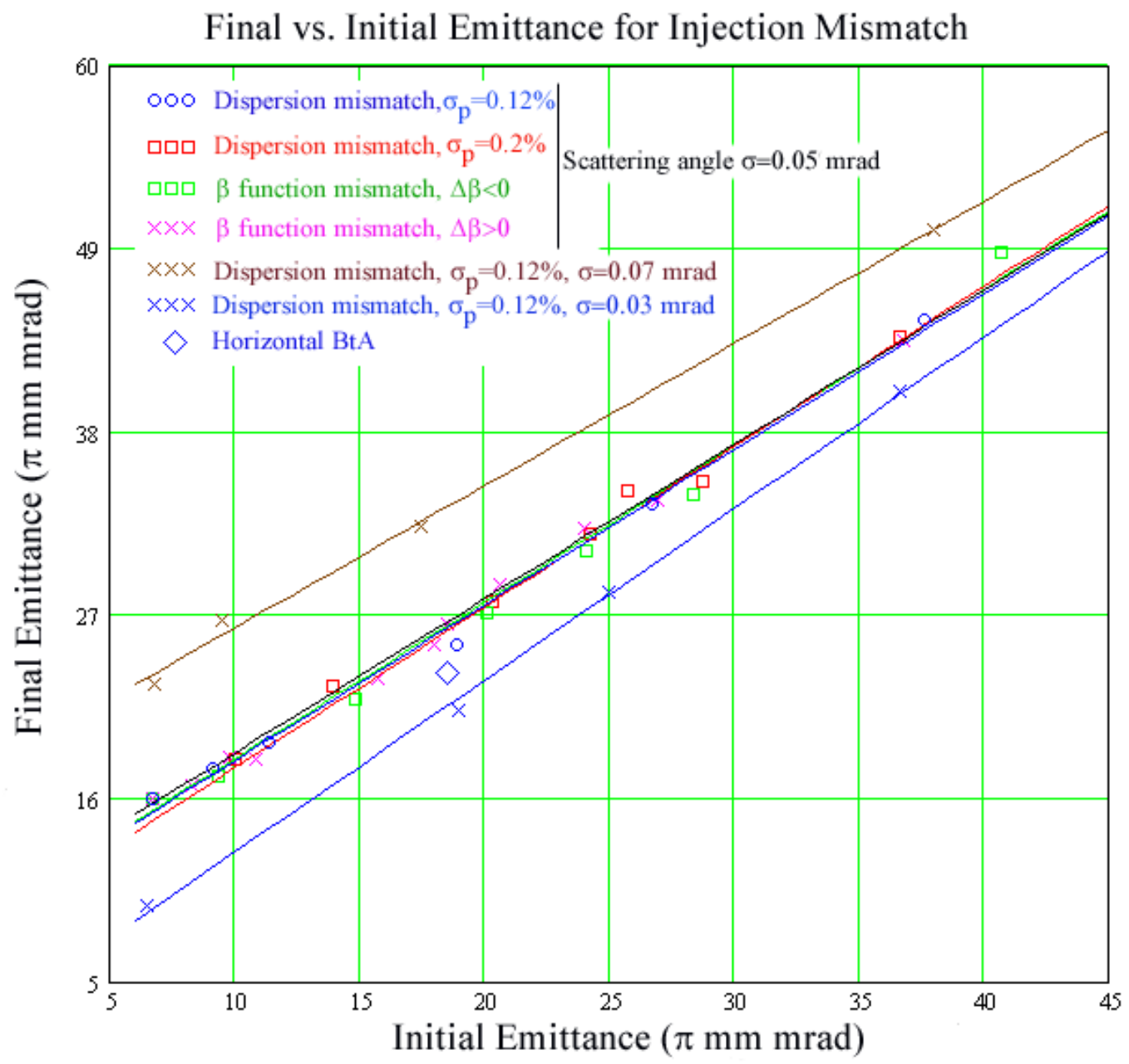

Figure 8: The effect of $\beta_{\mathrm{x}}$ and $\mathrm{D}$ mismatching on the Booster's Horizontal Emittance and Emittance Growth. Initial incoming (LtB) emittance is $6.89 \pi \mathrm{mm} \mathrm{mrad}$.

\section{Foil Scattering Angle Distributions}

The foil is composed of Carbon and its surface density is $200 \mu \mathrm{g} / \mathrm{cm}^{2}$. Scattering angle data exists expressed in terms of the number of particles per unit scattering angle, or 'angular density', as a function of scattering angle. ${ }^{4}$ More specifically, the angular

${ }^{4}$ From D. Raparia. 
density, $\rho(\varphi)$, is the number of particles that fall within a range from $\varphi$ to $\varphi+\Delta \varphi$, where $\Delta \varphi=1 / 360 \mathrm{mrad}$. This angular range corresponds to a disk of area $\Delta \mathrm{A}=2 \pi \mathrm{d}^{2} \varphi \Delta \varphi$, where $\mathrm{d}$ is the distance from the foil to the disk, and $\rho(\varphi)=\Phi(\varphi) \Delta$ A. So a Gaussian flux distribution $\Phi(\varphi)=\mathrm{C}_{1} \exp \left(-\varphi^{2} / 2 \sigma^{2}\right)$ corresponds to an angular distribution $\rho(\varphi)=C_{2} \varphi \exp \left(-\varphi^{2} / 2 \sigma^{2}\right)$, where $\Delta \varphi$ is a constant contained in the $C_{2}$ term. Figure 11 shows the distribution together with Gaussian distributions expressed as angular density vs. scattering angle.

$\varepsilon_{\mathrm{f}} / \varepsilon_{\mathrm{i}}$ as a function of Dispersion and $\beta$ Injection Mismatch for $\sigma=0.05 \mathrm{mrad}$

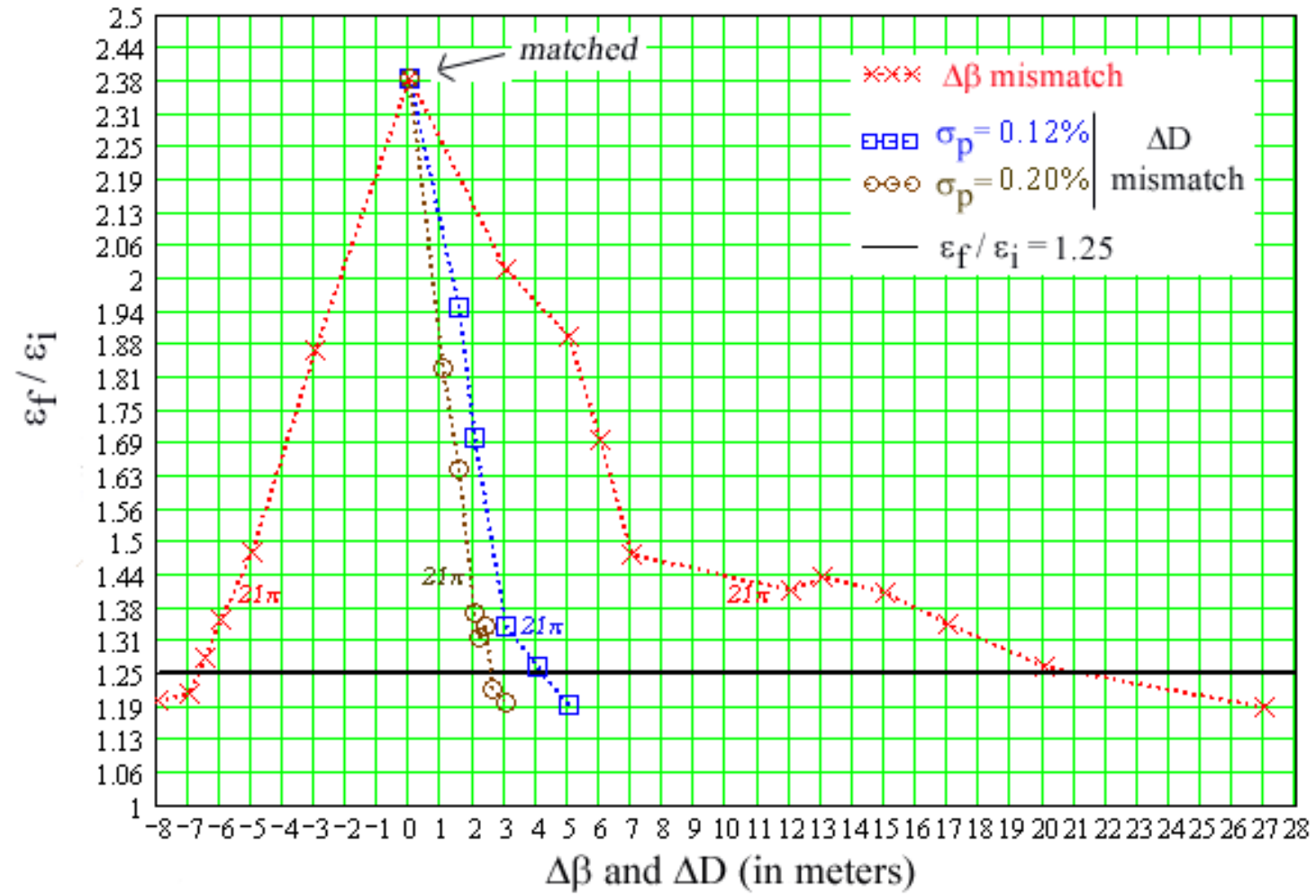

Figure 9: Horizontal 95\% normalized $\varepsilon_{f} / \varepsilon_{\mathrm{i}}$ as a function of $\beta$ and Dispersion $\left(\sigma_{\mathrm{p}}=0.12 \%\right.$ and $0.2 \%)$ mismatches. The approximate location where the average $\varepsilon,\left(\varepsilon_{\mathrm{f}}+\varepsilon_{\mathrm{i}}\right) / 2$, equals that observed in $\mathrm{BtA}$ is also marked for the 4 cases where the data intercepts it. Also shown is $\varepsilon_{\mathrm{f}} / \varepsilon_{\mathrm{i}}=1.25$, the amount of horizontal emittance increase seen in BtA. $\beta=10.85 \mathrm{~m}, \mathrm{D}=2.41 \mathrm{~m}$. Initial incoming (LtB) emittance was $6.89 \pi \mathrm{mm}$ mrad.

The first moment of $\varphi$ for the angular density distribution $\rho(\varphi)$ can be found from,

$$
\varphi_{1 s t}=\frac{\sum_{k=0}^{n} N_{k} \Delta \varphi}{\sum_{k=0}^{n} N_{k}}
$$

where there are $n$ data points. The maximum scattering angle considered is $\mathrm{n} \Delta \varphi=99 *(1 / 360 \mathrm{mrad})=0.275 \mathrm{mrad}$, and $N_{k}$ is the number of particles at the $k t h$ data point, or where $\varphi=k \Delta \varphi$. The value of $\varphi_{1 \mathrm{st}}$ obtained is $0.043 \mathrm{mrad}$. Considering flux distributions, $\sigma=0.05 \mathrm{mrad}$ corresponds to an angular density first moment of 0.063 
$\mathrm{mrad}, \sigma=0.03 \mathrm{mrad}$ to one of $0.038 \mathrm{mrad}$. A Gaussian distribution with $\sigma=0.034 \mathrm{mrad}$ has the same first moment as the expected distribution $(0.043 \mathrm{mrad})$.

$\varepsilon_{\mathrm{i}}$ vs. $\left(\varepsilon_{\mathrm{f}} / \varepsilon_{\mathrm{i}}\right)$ for $\sigma=0.03,0.05$, and $0.07 \mathrm{mrad}$

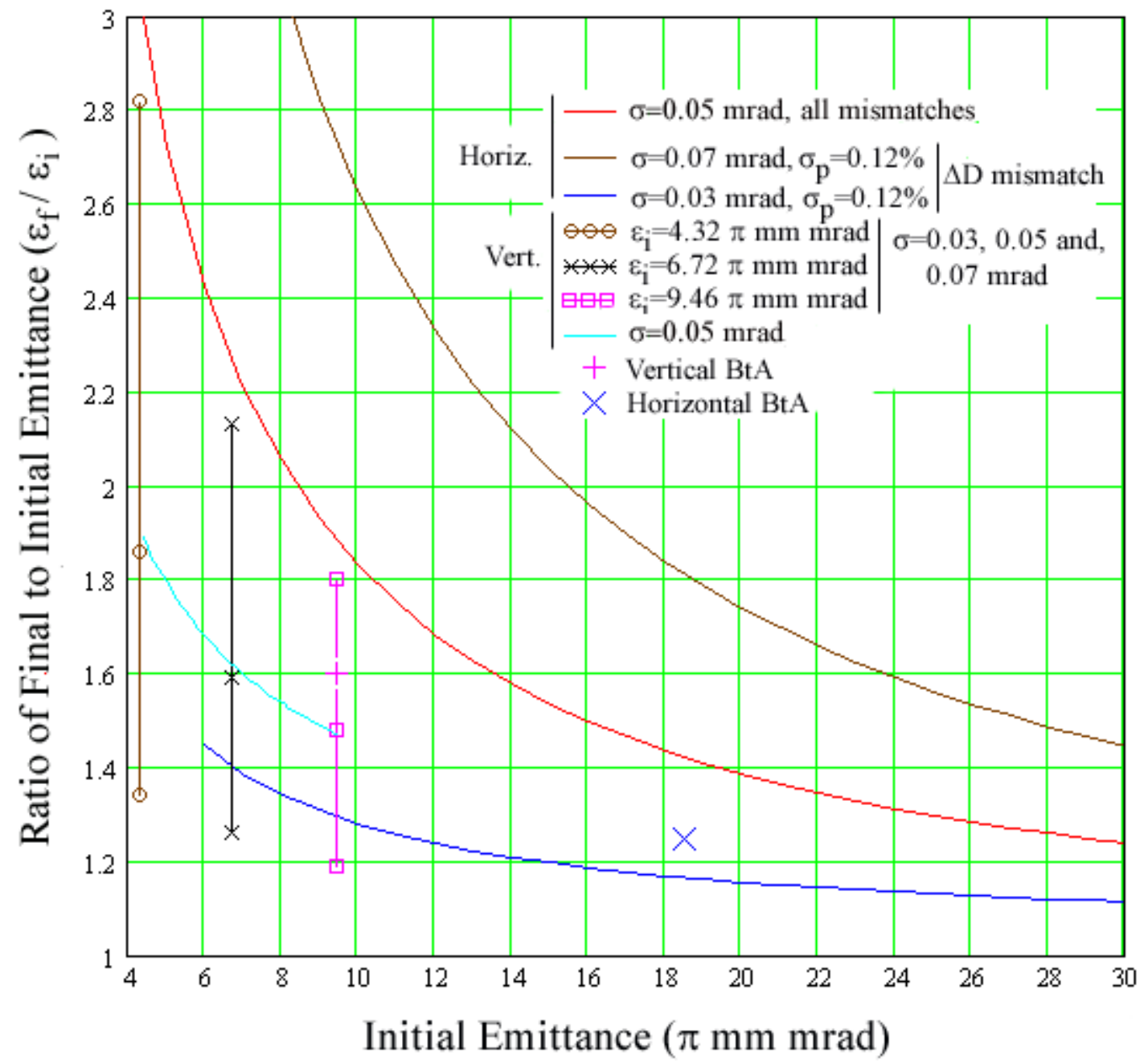

Figure 10: $\varepsilon_{\mathrm{f}} / \varepsilon_{\mathrm{i}}$ as a function of initial emittance for $\sigma=0.03,0.05$, and $0.07 \mathrm{mrad}$. The brown, red, and blue curves are derived from the linear fits of the data in figure 7 . The red curve is from a linear fit of all of the horizontal $\sigma=0.05 \mathrm{mrad}$ mismatch data. The three vertical lines on the left are the vertical data. Each line is for a different vertical $\varepsilon_{\mathrm{i}}$ (without mismatching). On each line, the lowest point is for $\sigma=0.03$, the middle point $\sigma=0.05$, and top point $\sigma=0.07 \mathrm{mrad}$. Both the horizontal and vertical BtA data is also shown. The cyan line is derived from a linear fit of the $\sigma=0.05$ vertical data (i.e.-the 3 middle points).

\section{Momentum Distributions}

The incoming beam's momentum distribution is typically taken more as parabolic, with a half width of about $0.33 \%$. An analysis similar to that done for the scattering distribution can be done for momentum distributions that are not Gaussian. For such a distribution, the first moment is $0.12 \%$. 


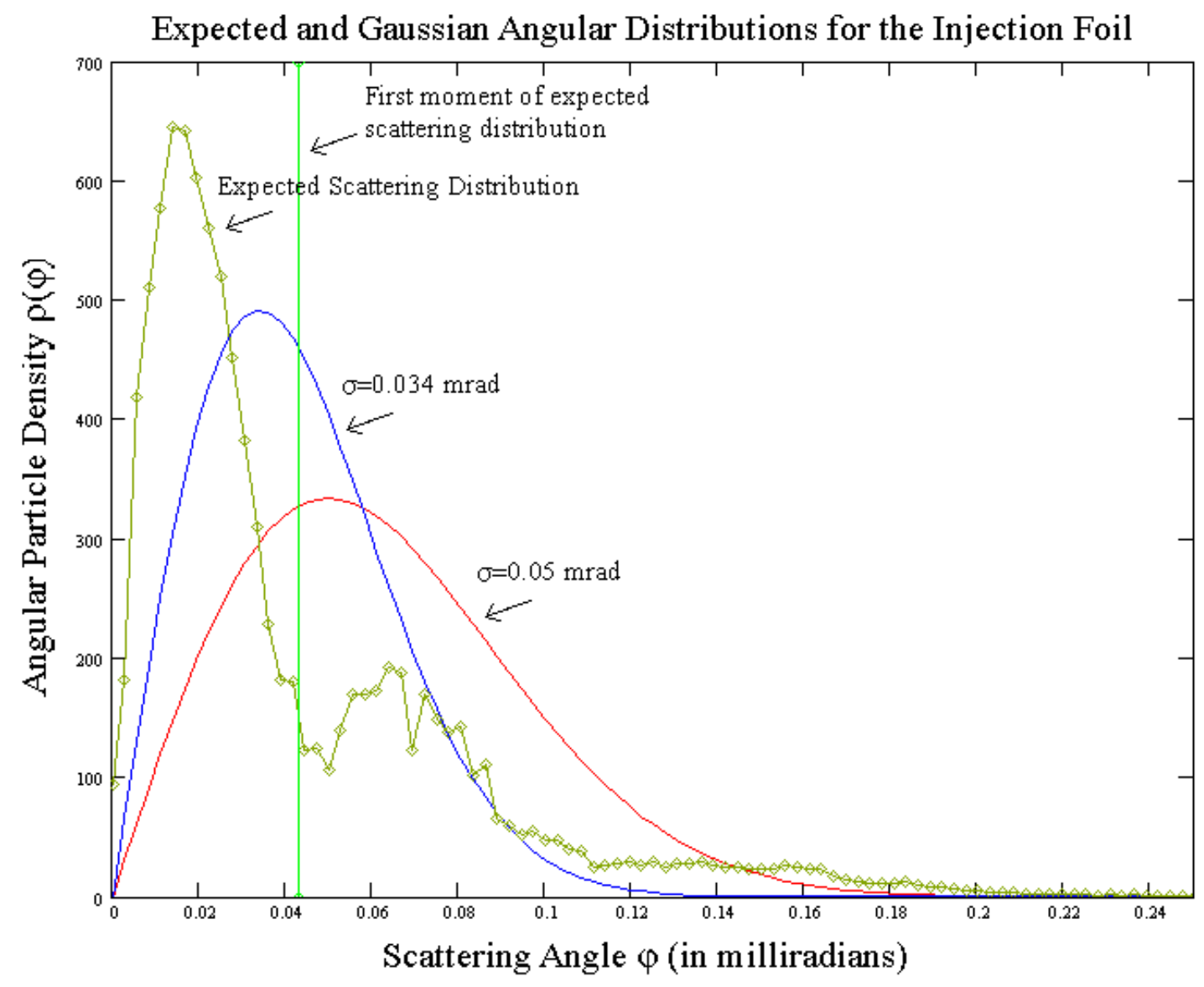

Figure 11: Angular distributions for expected and gaussian distributions of $\sigma=0.034$ and 0.05 mrad. All curves have the same area under their curves. Also included is the first moment of the expected scattering angle distribution $(0.043 \mathrm{mrad})$.

\section{Conclusion}

Data was taken to study the transverse emittance increase observed in BtA associated with the beam spending more time passing through the injection foil. Aside from some surprise at the amount of emittance increase that occurs due to passing through the foil, there are two outstanding issues that motivate the data taking and analysis. Firstly, why does the emittance increase more in the vertical plane than in the horizontal? Secondly, why is the horizontal emittance so much larger than expected?

It seems that both observations can be accounted for largely by a horizontal mismatch. A dispersion mismatch of $+3 \mathrm{~m}$ with $\Delta \mathrm{p} / \mathrm{p} \sigma=0.12 \%$ has fairly good agreement with the BtA data. A $\beta_{\mathrm{x}}$ mismatch of $-5 \mathrm{~m}$ or $+12 \mathrm{~m}$ also has fairly good agreement. However, there is still a discrepancy between the observations and particle tracking model: Assuming $\sigma=0.05 \mathrm{mrad}$, the horizontal emittance in BtA is not quite large enough to account for the lack of emittance increase from passage through the foil, and the vertical emittance in $\mathrm{BtA}$ is not quite small enough to account for the size of the emittance increase observed. Specifically, in the horizontal, a $\Delta \mathrm{D}=+4 \mathrm{~m}$ and $\sigma_{\mathrm{p}}=0.12 \%$ gives an $\varepsilon_{\mathrm{f}} / \varepsilon_{\mathrm{i}}$ of 1.26 and an $\varepsilon_{\mathrm{i}}$ 
of $26.7 \pi \mathrm{mm} \mathrm{mrad}$. Whereas, an $\varepsilon_{\mathrm{i}}$ of $18.7 \pi \mathrm{mm} \mathrm{mrad}$ is required for $\varepsilon_{\mathrm{f}} / \varepsilon_{\mathrm{i}}=1.25$. In the vertical, an $\varepsilon_{\mathrm{i}}$ of $6.9 \pi \mathrm{mm} \mathrm{mrad}$ gives an $\varepsilon_{\mathrm{f}} / \varepsilon_{\mathrm{i}}$ of 1.59 . Whereas, an $\varepsilon_{\mathrm{i}}$ of $9.5 \pi \mathrm{mm} \mathrm{mrad}$ and an $\varepsilon_{\mathrm{f}} / \varepsilon_{\mathrm{i}}$ of 1.59 is seen in BtA.

A $\beta_{\mathrm{x}}$ mismatch of $(14.7-10.852) \mathrm{m}=+4.85 \mathrm{~m}$, and a $\mathrm{D}$ mismatch of $(7.49-2.41) \mathrm{m}=5.08 \mathrm{~m}$ is indicated by an LtB MAD run using quadrupole strengths for the currents that were being used in LtB. However, when these values for $\Delta \beta_{\mathrm{x}}$ and $\Delta \mathrm{D}$ are put into the particle tracking model they give an unrealistically large value for the the initial emittance $(>40 \pi$ $\mathrm{mm} \mathrm{mrad}$ ) for the incoming LtB emittance value of $6.9 \pi \mathrm{mm} \mathrm{mrad}$ that was used. This is due in particular to the size of the dispersion mismatch, which by itself would give an $\varepsilon_{\mathrm{i}}$ of $37.6 \pi \mathrm{mm} \mathrm{mrad}$ and an $\varepsilon_{\mathrm{f}} / \varepsilon_{\mathrm{i}}$ of 1.19 .

The first moment of the scatterng angle distribution as a function of angular density was calculated from existing data (see figure 11). The result of $0.043 \mathrm{mrad}$ is smaller than the optimal result from the particle tracking of $0.063 \mathrm{mrad}$.

Given the premise that the horizontal $\varepsilon_{\mathrm{f}} / \varepsilon_{\mathrm{i}}$ is smaller than the vertical due to the mismatch in the horizontal plane, a shorter, rather than longer linac pulse should be used when tuning the horizontal match at injection. It's tempting to use a longer pulse because the profiles in BtA are better, but an improvement in the match will result in an increase in $\varepsilon_{\mathrm{f}} / \varepsilon_{\mathrm{i}}$, and as a result it will be harder to see. It may be that the vertical match is easier to obtain in part because the effect of the foil is less in that plane. Also, the use of a thinner $100 \mu \mathrm{g} / \mathrm{cm}^{2}$ foil, which will be available this year, may make it easier to obtain a better match in both planes by reducing the clouding effect that emittance blow-up has on the BtA profiles.

\section{Acknowledgements}

Thanks to Nick Luciano for obtaining foil scattering data and Kevin Brown for the LtB MAD model data. 